\title{
A synergetic layered inorganic-organic hybrid film for conductive, flexible, and transparent electrodes
}

\author{
Devendra Singh ${ }^{1}$, Ran $\operatorname{Tao}^{1}$ and Gilles Lubineau (D)
}

Conductive electrodes are major components of flexible optoelectronic devices. However, existing materials are either very conductive but brittle (e.g., ITO [indium tin-oxide]), or non-brittle but less conductive, with an environment-dependent conductivity (e.g., PEDOT:PSS [poly-(3,4 ethylenedioxythiophene): poly (styrene sulfonic acid)]). Here, we propose a new design that simultaneously takes advantage of both the high conductivity of ITO and the high flexibility of PEDOT:PSS. In our design, a PEDOT: PSS interface is inserted between the film substrate and the ITO layer, creating a hybrid layered structure that retains both its high conductivity and high stability, when the film is deformed. The rational behind the creation of this structure, is that PEDOT:PSS, used as an interface between the locally delaminated ITO layer and the substrate, substantially reduces the detrimental effects of cracks on the electrode's conductivity. These results open the path for a new generation of transparent electrodes in advanced flexible devices.

npj Flexible Electronics (2019)3:10; https://doi.org/10.1038/s41528-019-0054-4

\section{INTRODUCTION}

Creating transparent, flexible and stretchable electrodes has proven to be a challenge in electronics, including optoelectronics, solar cells, touch screens and displays, and smart wearable systems. $^{1-5}$ Among the highly conducting and transparent materials suitable for conductive and transparent electrodes, indium tin-oxide (ITO) is one of the most widely used materials, specifically in the display industry and photovoltaic markets. In addition to its high transmittance in the visible region and its low electrical resistivity, ITO possesses good chemical stability and is easily fabricated using currently available techniques. This makes it a good candidate for numerous key applications, including flat panel/liquid-crystal/electro-chromic displays, sensors, solar cells, thin film transistors, UV photo detectors, and laser diodes. ${ }^{6-9}$ Although natural sources of Indium are limited in nature and will, one day, be depleted, ITO films are still the first choice for relevant industries; this is because Indium possesses exceptional electronic properties and environmental stability. ${ }^{9}$ However, various alternatives are being considered, including conducting polymers, carbon allotropes or nanostructured material networks. However, these materials do not outmatch ITO, in terms of initial conductivity and environmental stability. ${ }^{10-16}$

Currently, high-quality ITO films are prepared using a great variety of deposition techniques including, but not limited to, vacuum evaporation, magnetron sputtering (DC and RF), molecular beam epitaxy, pulsed laser deposition, chemical vapor deposition, spray pyrolysis, sol-gel reaction, etc. ${ }^{17}$ Due to its superior controllability, high uniformity over large area substrates and high deposition rate, magnetron sputtering is the most widely used technique for thin film deposition. ${ }^{18,19}$ To obtain high quality uniform ITO films, most techniques require high deposition temperatures $\left(400^{\circ} \mathrm{C}\right.$ or higher) that make them unsuitable for the fabrication of polymer-based devices. Using flexible polymer substrates is only possible at low temperatures. ${ }^{12}$
Current trends in flexible electronics also raise the issue of brittle behavior for polycrystalline ITO films and its derivatives (InZnO, InZnAlO, InGaZnO, InZnSnO, etc.), which restricts their applications, in spite of their high transparency and low resistivity. ${ }^{6,8}$ Indeed, the development of channel cracks in the ITO layer, when stretched, has a detrimental effect on the electrical conductivity and optical transparency of the electrodes. ${ }^{19-24}$ Although this phenomenon has been known and frequently investigated, we have shown only recently that channel cracking does not directly affect the conductivity of the cracked electrodes. Rather than the cracking itself, it is, in fact, the delamination between the ITO layer and the substrate that changes the effective conductivity of the electrode. ${ }^{25}$ When the ITO layer is cracked, the electrical current can not flow inside the ITO layer from one fragment to the next due to the presence of the cracks. At the location of the cracks, the current can only flow within the substrate. If the distance between the fragments is very short, an efficient electrical bridging can still take place through the substrate reducing the effect of cracks on the effective conductivity. However, if delamination occurs between the ITO layer and the substrate, bridging cannot take place anymore, as the current cannot flow over a long distance in the highly insulating substrate. The delaminated area between the ITO and its substrate, and the electrical conductivity of this delaminated area, are then key in ensuring the integrity of the electrode with respect to channel cracks. Reducing delamination is primordial. If this is impossible, the delaminated area should be made as conductive as possible. ${ }^{25}$ This finding implies that engineering a higher conductivity interface on the delaminated area could largely reduce the detrimental effects of the cracks. This is what has set the rational for the work presented here.

Providing ITO films with the ability to both flex and stretch (bending cycling and tensile strain) would have tremendous impacts on the durability, performance, and stability of

\footnotetext{
'Physical Sciences and Engineering Division (PSE), COHMAS Laboratory, King Abdullah University of Science and Technology (KAUST), Thuwal 23955-6900, Saudi Arabia Correspondence: Devendra Singh (devendra.singh@kaust.edu.sa) or Gilles Lubineau (gilles.lubineau@kaust.edu.sa)
}

Received: 16 January 2019 Accepted: 11 April 2019

Published online: 08 May 2019 
transparent electrodes for the applications mentioned earlier on. Some of the ITO competitors may actually be used, in a synergetic way, to further improve the properties of the ITO/substrate interface. Due to their inherent flexibility, conductive polymers are good candidates for flexible electronics. ${ }^{26}$ They can sustain higher strains and large numbers of bending cycles before being damaging. However, they also show some important limitations. Their conductivities are usually smaller than those of ITO-based solutions, and they suffer from a poor environmental stability. Here, we focus our study on poly-(3,4-ethylenedioxythiophene) (PEDOT), doped with poly-(styrenesulfonic acid) (PSS) [thus PEDOT:PSS], which serves as counter-ion for the positively charged PEDOT. PEDOT:PSS has emerged as a good conductive polymer, due to its high conductivity and overall performance among other alternatives in aqueous form. ${ }^{18,21}$ Its conductive performance can be significantly improved by using solvents. Indeed, ethylene glycol (EG) or dimethyl sulfoxide (DMSO), for example, produces a rearrangement of the morphology of the films, thus promoting a phase separation between the conducting PEDOT and the insulating PSS. This leads to a better conducting network, and can even change the work function of the film. ${ }^{26-28}$ Large increases in conductivity have been reported when using a polar solvent such as EG. ${ }^{29}$ Due to its highly hygroscopic nature, PEDOT: PSS's behavior is temperature-dependent and moisture-dependent, which results in degraded properties in case of exposure to the environment.

In this work, our objective is to create a new type of transparent electrodes that would benefit both from the high conductivity of ITO and from the high flexibility of PEDOT. We have then merged the valuable properties of ITO (conductive, transparent, robust in harsh environment) and of doped PEDOT:PSS (conductive, transparent, flexible). We propose a synergetic layered structure obtained from the sputtered ITO film, together with an intermediate (or buffer) layer of EG-doped PEDOT:PSS on the PET substrate for potential flexible optoelectronic applications. Using PET as a common substrate, we have built and performed comparative studies using different types of electrodes with the following structures: PEDOT:PSS on PET (now onwards PP), ITO on PET (now onwards IP), and ITO on PEDOT:PSS on PET (now onwards IPP). For each structure, the effects from annealing have also been quantified. The results presented below demonstrate that the hybrid IPP structure, in which a very thin PEDOT:PSS buffer layer is introduced at the ITO/PET interface, possesses interesting properties, somewhat "in-between" those of the PP and IP single-layer structures.

\section{RESULTS AND DISCUSSION}

Cleaned PET sheets ( $250 \mu \mathrm{m}$ thick) were used as base substrate to deposit the required consecutive layers. Process parameters of the RF magnetron sputtering technique are optimized in order to obtain highly conducting and transparent ITO thin films deposited (thickness $\sim 100 \mathrm{~nm}$ ) on the different layered substrates, at room temperature. Depending on the sample, the optimized ITO film is deposited either directly on the PET substrate, or on a buffer PEDOT:PSS layer that is beforehand deposited on the PET substrate.

To obtain the buffer layer, a highly conductive polymer was prepared by magnetic stirring ( $500 \mathrm{rpm}$ for $6 \mathrm{~h}$ ) of an aqueous dispersion of PEDOT:PSS Clevios PH1000 doped with 3 wt.\% of a polar solvent (EG in our case). EG was used to enhance the electrical conductivity of the PEDOT:PSS solution and the weight content of EG in the PEDOT:PSS dispersion was optimized in a previous work. $^{29}$ This EG-doped PEDOT:PSS solution was then spin-coated to obtain the buffer layer with a thickness $\sim 50 \mathrm{~nm}$. This thickness was used as it corresponds to the minimum thickness achievable while preserving uniformity. This is sufficient to demonstrate the bridging effect of the PEDOT:PSS layer that is our objective in this publication. To obtain a uniform distribution of the spin-coated EG-doped PEDOT:PSS layer, we first grew a few layers $(\sim 5 \mathrm{~nm})$ of hydrophilic 3-aminopropyltriethoxysilane (APTES) on the PET substrate, using a molecular vapor deposition (MVD) technique. The adsorption of the APTES layer (likely through hydrogen bonding by the amine) to the polymer substrate (PET in this case) helps the formation of lateral bonds which, in turn, help the formation of a multilayer via adhesion. ${ }^{30,31}$ A schematic representation of the step-by-step layer deposition on the PET substrate is shown in Fig. 1.

The crystalline structure (i.e., size and orientation of the grains) was determined by the analysis of the X-ray diffraction (XRD) measurements, for the $2 \theta$ range of $10-55^{\circ}$ (Fig. 2a). The XRD patterns obtained for the as-prepared film confirm the uniform growth of the ITO-deposited layer (JCPDS 17-2194). Grain sizes for ITO films deposited on different substrates were estimated using the Scherrer formula $(d=0.9 \lambda / \beta \cos \theta)$, where $d$ is the crystallite size, $\lambda$ the wavelength of the X-rays, $\beta$ the full width at halfmaximum, and $\theta$ is the Bragg angle. ${ }^{32}$ The estimated crystallite size, calculated with preferred (411) orientation, was found to be $\sim 48 \pm 2 \mathrm{~nm}$. Typical XRD patterns of sputtered ITO thin films in IPlayered and IPP-layered structures showed a reflection with sharp peaks at $\sim 37.65^{\circ}$ and $\sim 45^{\circ}$, which correspond to the preferred orientations of (411) and (431) planes of ITO, respectively.

The specular optical transmittance for the various sets of samples in the wavelength range of $300-800 \mathrm{~nm}$ are shown in

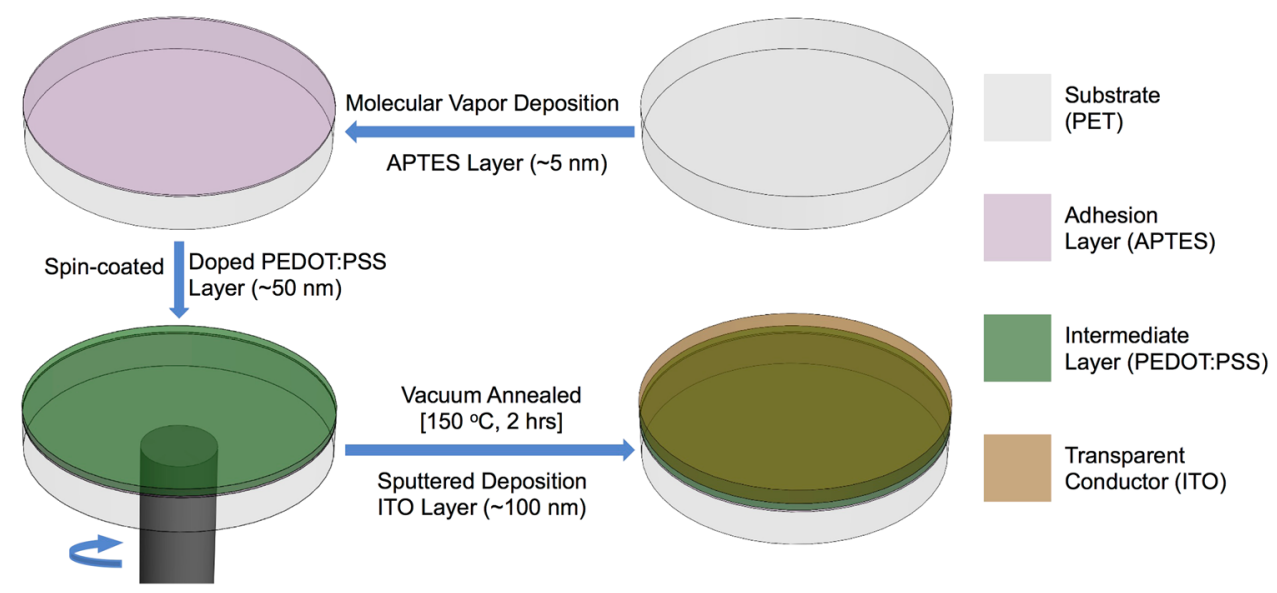

Fig. 1 Schematic of step-by-step deposition process of sputtered ITO films on spin-coated EG-doped PEDOT:PSS layer on APTES-treated PET substrate 

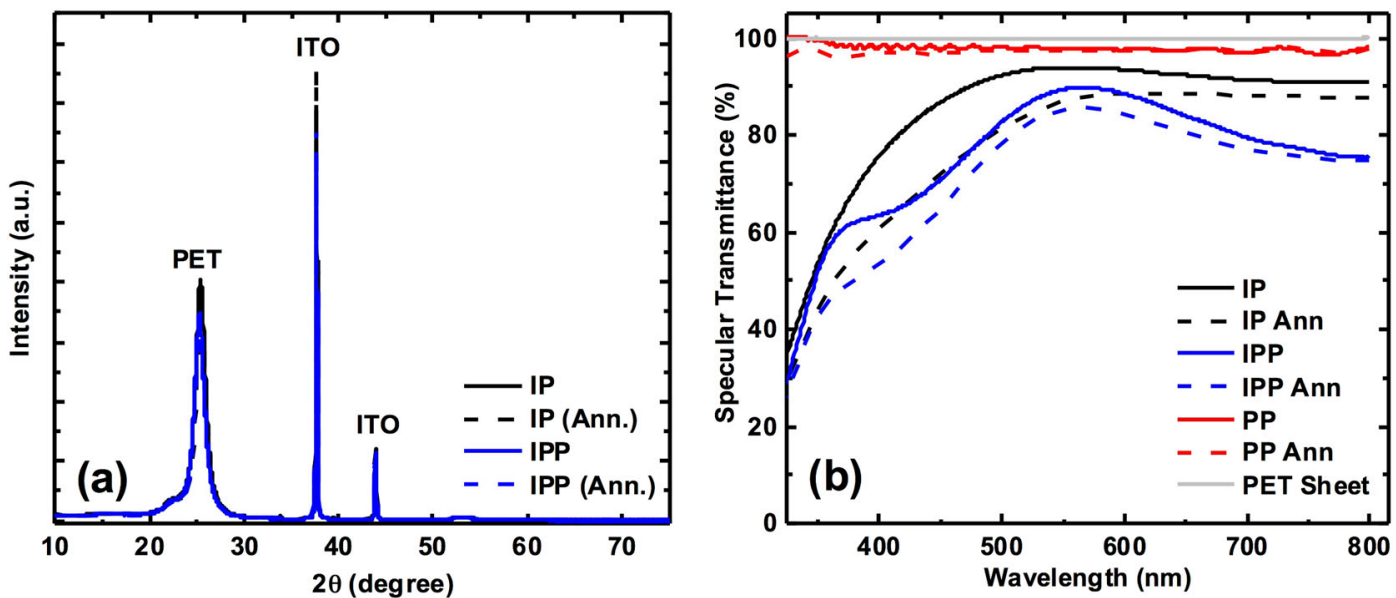

Fig. 2 (a) X-ray diffraction of various sputter-deposited ITO films on PET substrate (as-deposited and annealed), with and without EG-doped PEDOT:PSS interlayer (shown in figure), and (b) Specular transmittance spectra of films deposited on various sample sets (mentioned in figure)
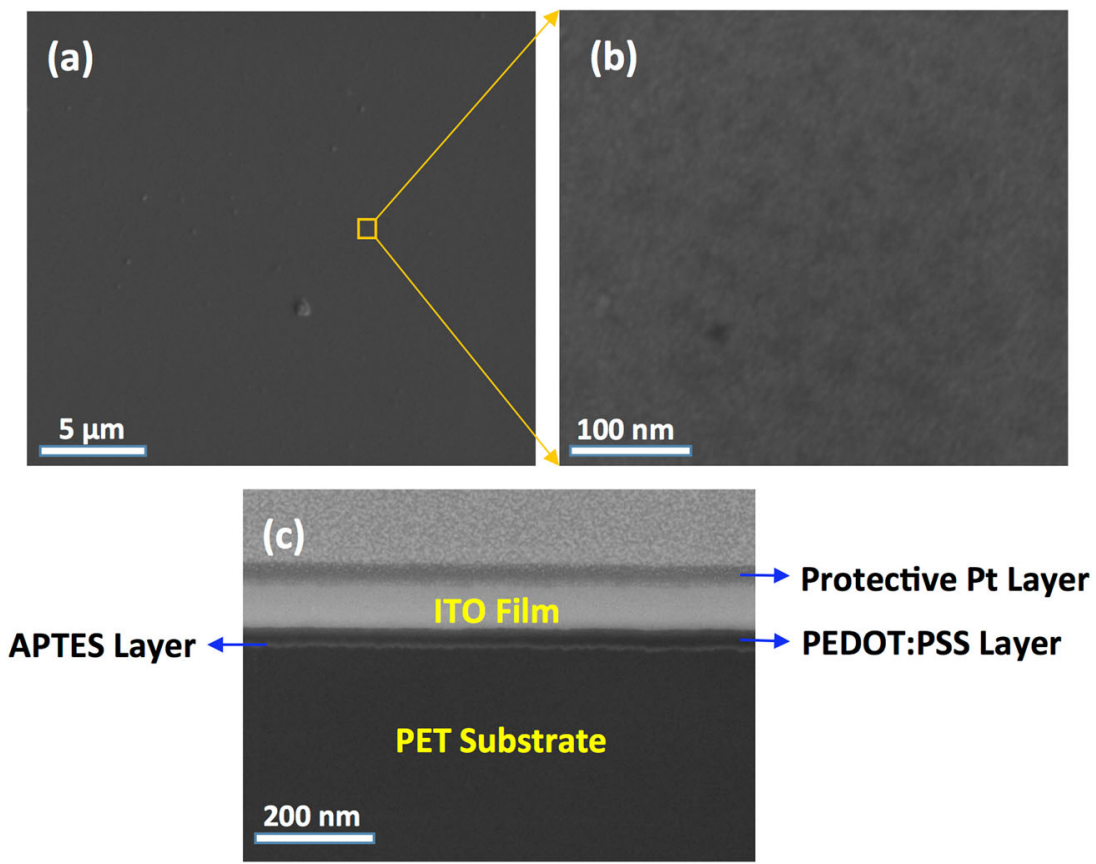

Fig. 3 SEM images of ITO coated on PEDOT:PSS on PET substrate, top surface image with (a) low resolution and (b) high resolution images, and (c) high resolution cross-sectional image of IPP stacked layer (scale and involved layers are shown in respective figures)

Fig. 2b. Results show that the average transmittance values are, asexpected, dependent on the number of layers and materials involved. The transparency of the hybrid IPP structure is very competitive in the wavelength range of $300-600 \mathrm{~nm}$. Its transparency decreases by $10 \%$, when compared with ITO at higher wavelengths $(600-800 \mathrm{~nm})$, but is still reasonable for most applications. The haze value for our prepared samples is not reported, as it is consistently less than $2 \%$ for all samples. Low haze is requested for applications that require high transparency and clarity (e.g., flexible displays and touch screens). Our samples, which feature both low haze (less than 2\%) and high transparency (more than $85 \%$ measured at a wavelength of $550 \mathrm{~nm}$ ), are thus good candidates for such applications.

Microscopic studies were also carried out to observe the surface morphology of the sputter-deposited ITO films on a typical PP structure. Figure 3 shows Scanning Electron Microscope (SEM) images of a typical sample of sputtered ITO thin film deposited on PEDOT:PSS on PET substrate, at room temperature: (a) low resolution images, (b) high resolution images, and (c) high resolution focused ion beam FIB/SEM cross-sectional image of IPP stacked layer (involved layers are shown in respective figure). All the samples show a uniform distribution of grains through SEM imaging.

Electrical resistance response of strained thin films

We have investigated the change in electrical resistance of the film when discrete degradations such as channel cracks and associated delamination are introduced. The experimental protocol involved stretching the films in a tension mode to introduce a quasi-periodical pattern of cracks, while monitoring the change in electrical resistance so it can be correlated to the change in strain and to the change in crack density. Electrical resistance was first measured on the loaded film, using an in-situ technique (Fig. 4). This corresponds to an "open-crack" configuration due to the tension in the film. Then, we measured the residual electrical resistance after unloading the film (Fig. 5) to evaluate the eventual 

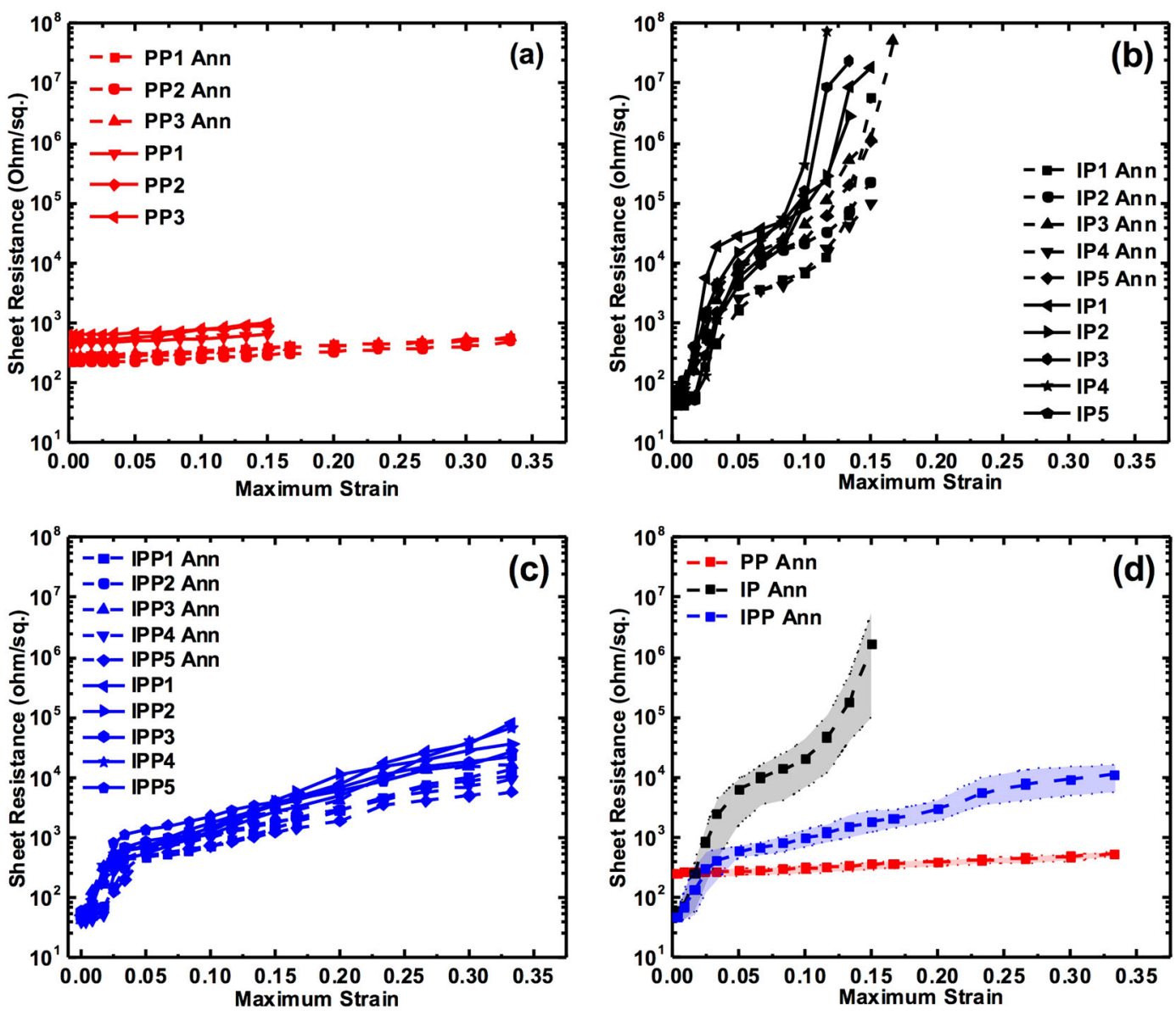

Fig. 4 Applied strain versus two-probe in-situ sheet resistance (monotonic tensile testing), for the following sets of films: (a) PP, (b) IP, and (c) IPP, for both as-deposited and annealed samples. Comparison of the envelopes for PP, IP, and IPP annealed samples to ease the comparison (d)

effects of cracks closure. These measurements could later be correlated to the crack density by counting the cracks on microscopic images. All in-situ microscopic images of the various specimens were captured under controlled applied micro-tensile strain, using a Kammrath and Weiss frame. In-situ SEM images acquired during the micro-tensile testing of typical IPP stacked structure (annealed) are shown in Fig. 6.

For the monotonic tensile loading, we measured the in-situ electrical resistance with respect to applied strain and results are shown in Fig. 4 for various set of deposited films as following (a) PP, (b) IP, and (c) IPP. For each layered structure, the results are presented for both non-annealed (solid line) and annealed films (dashed lines). Figure $4 d$ is a superposition of the envelopes of all configurations to ease the comparison.

When it comes to the initial resistance before any loading, the as-deposited PP layers (which conductivity only relied on PEDOT: PSS) have a relatively high initial sheet resistance $(\sim 500-700 \mathrm{Ohm} /$ sq). Further vacuum annealing of the samples, at $150^{\circ} \mathrm{C}$, for $2 \mathrm{~h}$, results in a slight improvement in the electrical sheet resistance $(\sim 200-400 \mathrm{Ohm} / \mathrm{sq})$, but the initial resistance is always much lower for ITO containing samples. As-deposited and annealed IP samples have a significantly lower sheet resistance $(\sim 50-60 \mathrm{Ohm} / \mathrm{sq})$. The hybrid IPP (both as-deposited and annealed) samples also feature an excellent initial resistance around $40-60 \mathrm{Ohm} / \mathrm{sq}$. So, before any loading, the IPP samples have an electrical performance similar to the one of highly conductive ITO based samples (IP).

When it comes to the evolution of the sheet resistance during mechanical loading, the sheet resistance of PP samples is very stable as it only increases by $50 \%$ between 0 and $15 \%$, and $100 \%$ between 0 and $30 \%$ strain. On the contrary, the sheet resistance of the IP samples increases drastically as soon as the macroscopic strain resulted in the degradation of the structure: by $10^{7} \%$ between 0 and $15 \%$, and above measurable values between 0 and $30 \%$. The samples experienced the expected type of degradation pattern in such laminated systems (illustrated on Fig. 6 on IPP samples that experience similar degradation morphology). ${ }^{23} \mathrm{At}$ low strains, channel cracks run perpendicular to the loading direction and tend to form a quasi-periodical network with the increasing crack density. At higher strains, Poisson's effect induces transverse contraction, resulting in localized buckling and delamination. Previous studies have shown that the presence of delamination at a very early stage in the loading (due to a concentration of the stress at the crack tips) is the main mechanism responsible for the degradation of electrical performance. ${ }^{23}$ The hybrid IPP (both as-deposited and annealed) samples also feature an increase in resistance with the development of cracks, but the amplitude of this increase is much lower. The sheet resistance of IPP samples increases by $2000 \%$ between 0 and $15 \%$, and $2 \times 10^{4} \%$ between 0 and $30 \%$ strain. These are large value but very limited compared with the value of pure ITO (IP) based samples. We also highlight that in the case of the PP and IPP structures, we do not observe transverse cracks in the PEDOT/PSS layer.

Similar conclusions can be drawn for measurements on unloaded films. However, due to the partial closure of the cracks after unloading, the measured resistance is always lower than that for loaded samples (Fig. 5). Figure 5(d) illustrates a stretched ( 10\%) IPP based sample being highly bended performing with a light-emitting diode (LED) (inset image shows the LED glow without any bending to the IPP sample). 

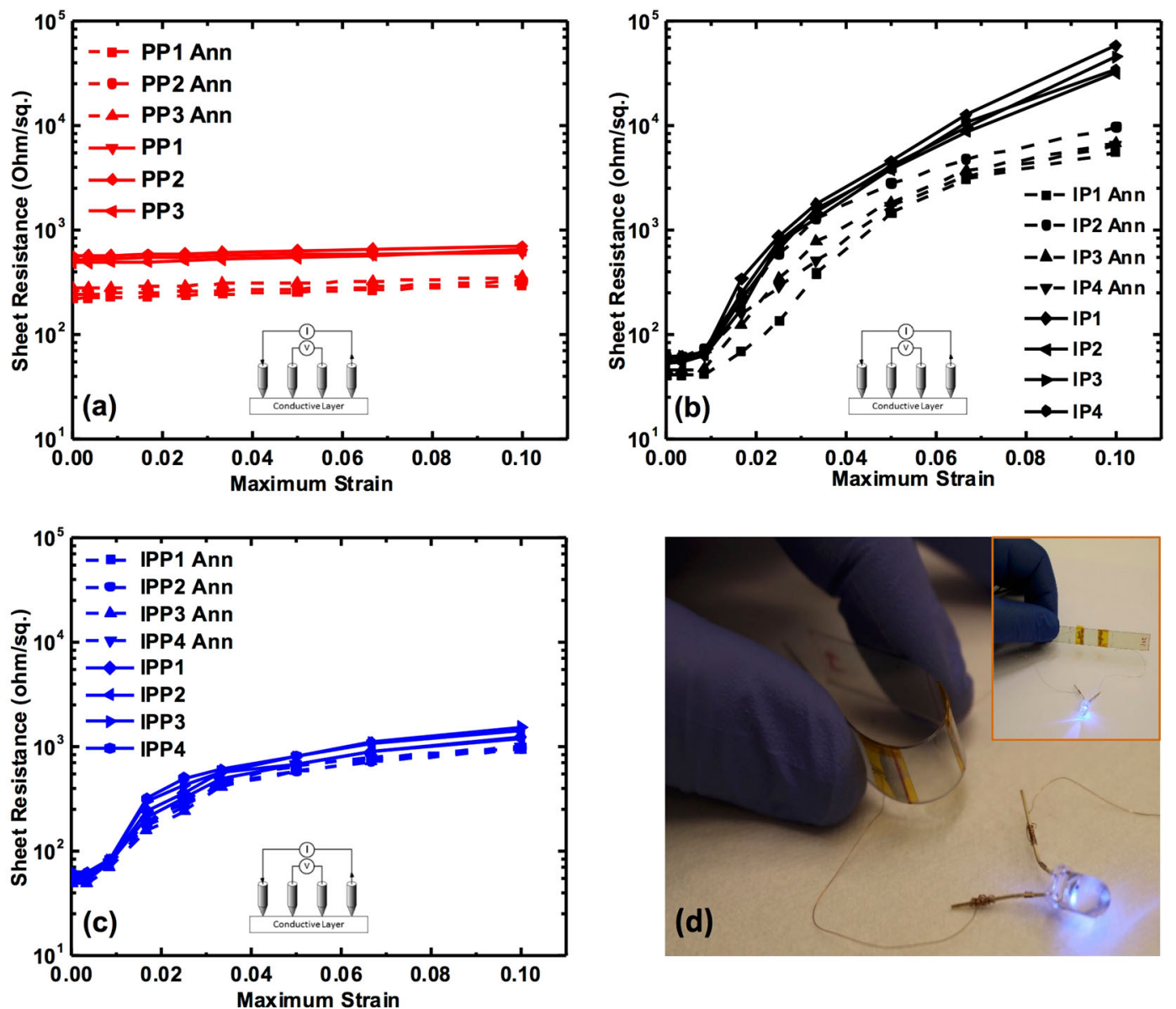

Fig. 5 Applied strain versus four-probe sheet resistance (loading/unloading tensile testing), for the following sets of films: (a) PP, (b) IP, and (c) IPP, respectively, for both as-deposited and annealed samples. d Photograph showing a stretched ( 10\%) IPP based sample being highly bended performing with a LED, inset shows the LED glow without bending the IPP sample

The ability of the intermediate conductive layer to improve the stability of the film's conductivity when cracking appears is here very obvious. Combining ITO with PEDOT in a composite with a layered structure results in a hybrid behavior characterized by high initial conductivity and better stability with strain.

\section{Effect of channel crack density}

The change in electrical resistance in layered structures is associated mainly with the multiplication of transverse cracks that trigger delamination between the conductive ITO and the substrate. Therefore, it is important to examine the dynamics of the multiplication of cracks in the various layered structures. The advantage of SEM is that it offers very good contrast, which makes it possible to easily observe the characteristic features of the cracked pattern. Figure 6 clearly shows the multiplication of wellpercolated channel cracks that give birth to secondary cracks, when the strain is significant. To follow the multiplication of cracks in-situ, these were observed during the monotonic tensile tests presented above using optical microscopy. The corresponding digital images were used to both track the number of cracks during the test and evaluate the applied strain over the region of interest (details provided in Figs. S1 and S2, Supplementary Information). To quantify the crack spacing with respect to the coating thickness, we define the dimensionless channel cracking rate, $\rho$, as $\rho=h_{c} / L$; where $h_{c}$ is the coating layer thickness and $L$ is the average inter-crack spacing, which is equal to the length of the region of interest (ROI) over which the cracks are counted and then divided by the number of cracks. Figure 7 shows the change in channel cracking rate versus the average strain, for various sets of films referred as (a) IP layers and (b) IPP layers, respectively, for various sets of as-deposited and annealed samples. We found that the effect of annealing on the channel-cracking rate was very limited, whether we used as-deposited or annealed samples. Interestingly, the channel-cracking rate of IP-layered structures was found to be $25 \%$ higher than that of the IPP-layered structures. The intermediate PEDOT:PSS layer has then a second beneficial effect on the performance of the electrode. In summary, the first beneficial effect of the intermediate PEDOT:PSS layer is a reduction of the sensitivity of the electrical resistivity to the cracks (see Electrical resistance response of strained thin films section). The second beneficial effect is that, by introducing a soft interfacial layer, it slightly reduces the multiplication of cracks when a mechanical loading is applied. This can be attributed to the modification of the shear stress transfer at the interface.

Environmental stability studies

It is known that ITO and PEDOT:PSS have totally different responses to the environment (ITO being very stable, whereas PEDOT:PSS's electrical response can vary greatly). We show here that the hybrid IPP-layered structure also features interesting environmental stability.

After preparation following the protocol described in the experimental section, samples were placed inside a dedicated humidity and temperature control chamber controlled at $80 \%$ and $50^{\circ} \mathrm{C}$, respectively, with an exposure time of two hours. The exposure time was chosen to ensure the stabilization of the 

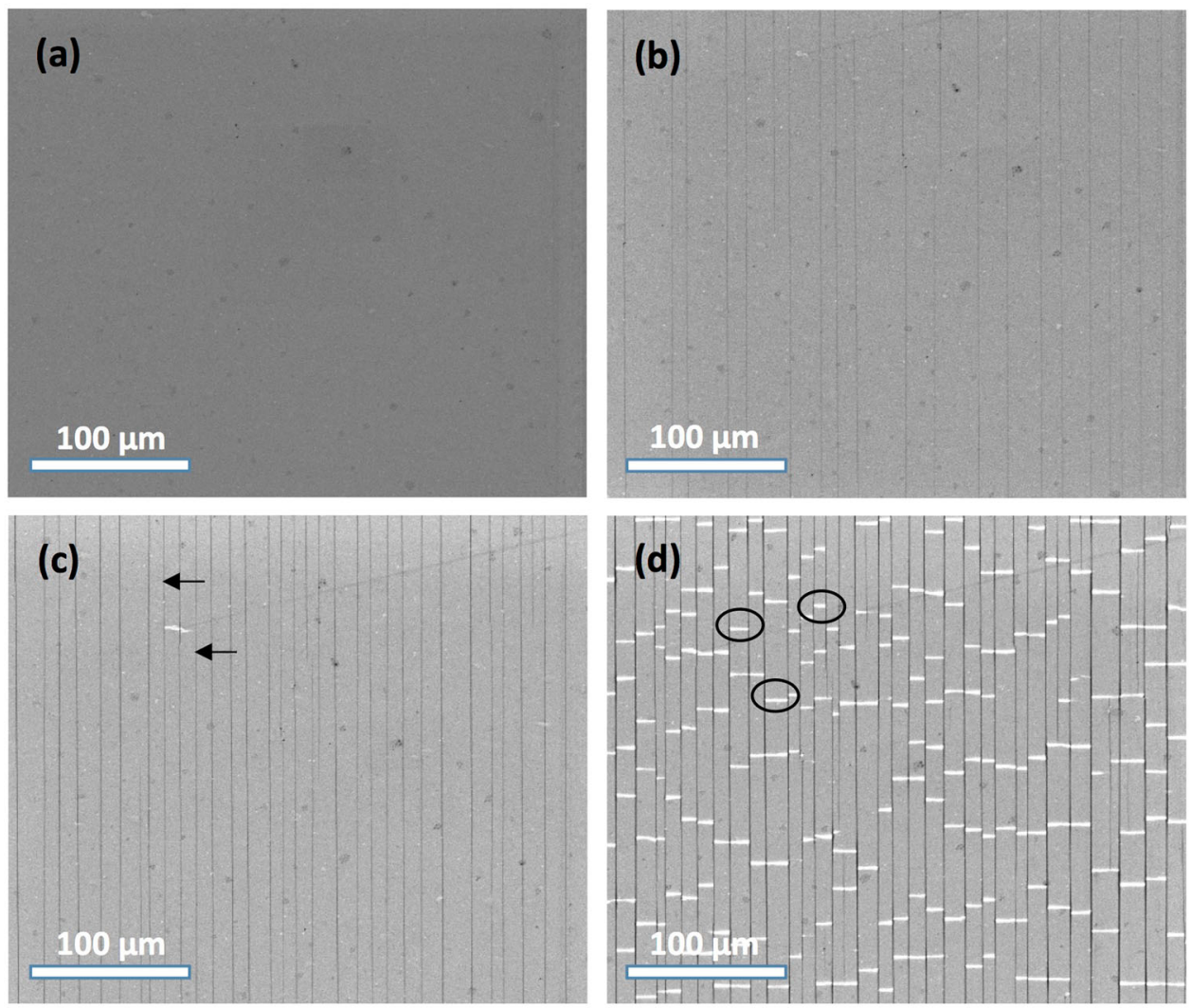

Fig. 6 In-situ SEM images acquired during micro-tensile testing of a typical IPP stacked structure. Average strain values prescribed on the IPP structure are: (a) $1.67 \%,(\mathbf{b}) 3.33 \%$, (c) $6.67 \%$, and (d) $10 \%$, respectively. Arrows: channel cracks, circles: secondary cracks and localized buckling

moisture uptake in the PEDOT:PSS phase. Indeed, both electronic and ionic conductivity performances of PEDOT:PSS depend on the moisture uptake. A preliminary experimental campaign on our EGmodified PEDOT/PSS demonstrated that 50 microns thin films of bulk PEDOT:PSS reach a stable moisture uptake after $1000 \mathrm{~s}$ (Fig. S3). Considering the thickness of the PEDOT:PSS interface used here $(50 \mathrm{~nm})$, a $1-\mathrm{h}$ exposure time is then largely enough to evaluate the change in electrical performance of the film when the PEDOT:PSS layer close to the cracks experiences moisture. The electrical sheet resistance was measured before and after exposure.

Figure 8 shows the relative change in sheet resistance for PP, IP, and IPP samples after exposure. For each sample, we have studied configurations with and without annealing and with and without pre-strain. The numerical data are provided in Table S1, supporting information. The following conclusions can be drawn for each type of material. PP samples are as expected very sensitive to moisture and experience a relative change in resistance between 14 to $23 \%$ depending on the configuration. On the contrary, IP samples are very stables and the resistance increases only between 0.6 to $1.5 \%$. Finally, the hybrid IPP samples experience a relative increase in resistance between 2.2 to 6.2. From these observations, we conclude that a layered synergetic IPP structure has a much more stable sheet resistance when humidity and temperature vary.

In this work, we present a new design based on a conductive polymer-assisted transparent and conducting ITO layer on a flexible substrate. Highly conductive and transparent sputtered ITO films on flexible PET substrates are prepared, either with or without an intermediate layer of PEDOT:PSS. They are then compared, in terms of their potentials for stretchable electrodebased applications. The brittle intrinsic nature of ITO layers makes them unsuitable for their use in flexible and stretchable devices, while the as-deposited PEDOT:PSS layers are prompt to the environmental degradation in atmosphere. Our new design counterbalances the limitations of both materials. Our studies show that, for a range of macroscopic strain values up to $30 \%$ the hybrid structure features a low initial resistivity and a high stability, when subjected to mechanical strains. This can be attributed to an improvement of the electrical transfer at the delaminated interfaces, due to the presence of the conductive PEDOT:PSS layer. This PEDOT:PSS layer also has a beneficial effect on the degradation kinetics, as the channel cracking density tends to be lower in the hybrid structure, compared with the ITO-only structure. An explanation for this is the change in mechanical load transfer at the interface, due to the presence of this soft layer. We also show that, when different sets of samples (PP, IP, and IPP layers, with and without pre strain, and for both as-deposited and annealed samples) are exposed to a harsh environment $(80 \%$ relative humidity and $50^{\circ} \mathrm{C}$ temperature), the electrical sheet resistance increases for PP structures, whereas that of IP and IPP layered structures does not change significantly. Results show that an integration of the highly conductive ITO layers with conducting polymer layers of PEDOT:PSS can be used as transparent electrodes in advanced stretchable and flexible devices.

\section{METHODS}

The poly-ethylene terephthalate (PET) sheet (thickness $250 \mu \mathrm{m}$ ), used as base substrate, was purchased from Goodfellow Suppliers, UK. The PEDOT: PSS aqueous dispersion (Clevios PH1000) was purchased from HC Starck, Inc. Ethylene glycol (EG), isopropyl alcohol (IPA) and acetone were purchased from Sigma-Aldrich. To sputter the ITO thin films, a copper back-plated ITO target (InO:SnO::90:10, diameter of 2 inch with $99.99 \%$ purity) was purchased from MaTeck Material Technologies \& Kristalle (GmbH, Germany). 

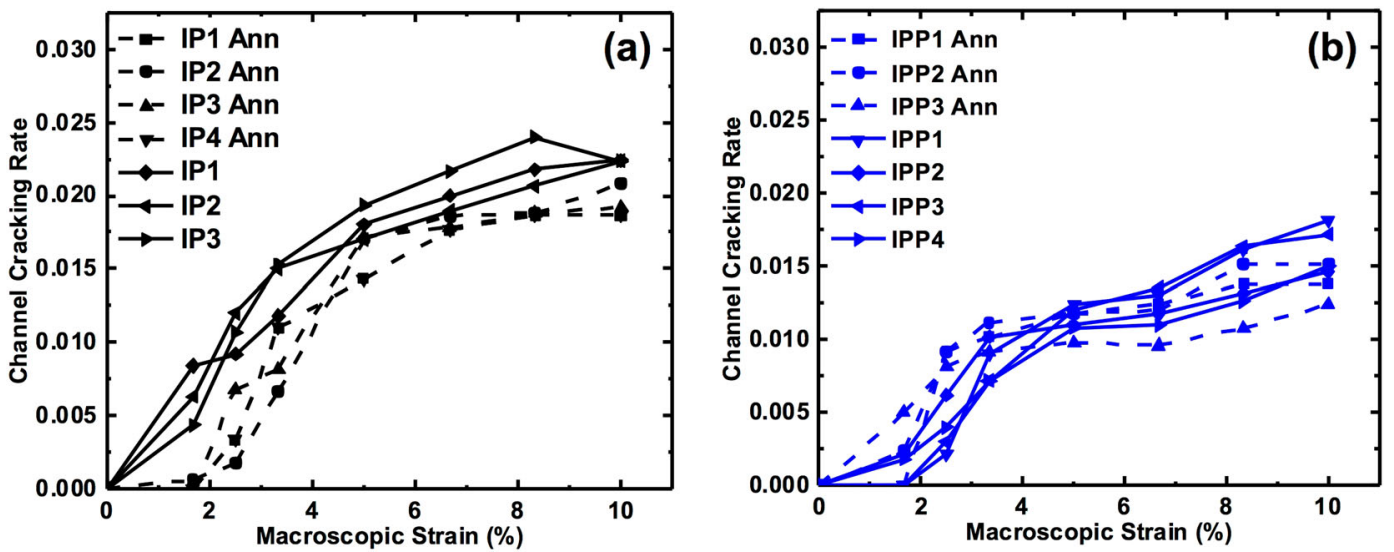

Fig. 7 Change in channel cracking rate with respect to the average macroscopic strain, for various sets of films ((a) IP and (b) IPP), as-deposited and annealed samples

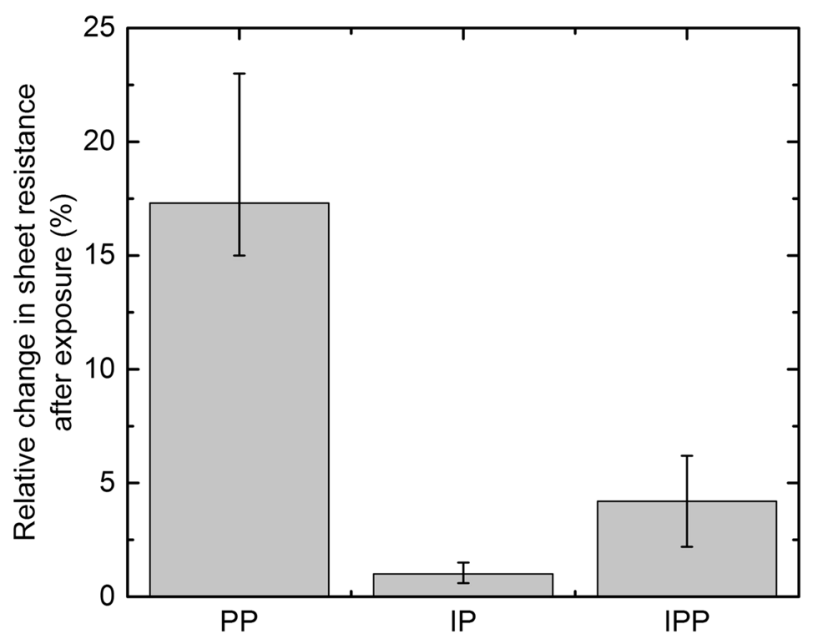

Fig. 8 Variations of average sheet resistance after exposure to environment $\left(80 \% \mathrm{RH}, 50^{\circ} \mathrm{C}\right)$

PET substrates were cleaned by sonication, for 5 min each, in IPA and deionized water, subsequently; then dried with nitrogen gas, followed by heat treatment, at $120^{\circ} \mathrm{C}$, in air (Memmert oven chamber).

Because of its simple structure and low cost, we chose the molecular vapor deposition (MVD) [AMST-100E] technique to deposit the hydrophilic layer of APTES (3-aminopropyltriethoxysilane). An $\mathrm{O}_{2}$ plasma treatment was performed at $200 \mathrm{~W}$, with an oxygen content of $200 \mathrm{sccm}$, for $100 \mathrm{~s}$ (inside MVD tool). To obtain a few layers of APTES $(\sim 5 \mathrm{~nm})$, the chamber pressure was kept at $4 \mathrm{mTorr}$ and the temperature at $35^{\circ} \mathrm{C}$. To obtain the buffer layer of highly conductive PEDOT:PSS, an aqueous dispersion of PEDOT:PSS Clevios PH1000 with 3 wt.\% of an ethylene glycol (EG) polar solvent was blended at $500 \mathrm{rpm}$, for $6 \mathrm{~h}$, using magnetic stirring. ${ }^{27}$ APTEScoated PET substrates were immediately spin-coated with an as-prepared EG-doped PEDOT:PSS solution (speed of $5000 \mathrm{rpm}$, for $30 \mathrm{~s}$ ) to obtain $\sim 50 \mathrm{~nm}$ thin layers.

High quality ITO thin films $(\sim 100 \mathrm{~nm})$ were deposited on desired substrates at room temperature, using a RF magnetron sputtering system (AJA Advanced Energy Tools Inc.). Optimal deposition conditions were found to be at a sputtering power of $60 \mathrm{~W}, 3 \mathrm{mTorr}$ sputtering pressure, $25 \mathrm{sccm}$ of Argon gas flow, with a $7 \mathrm{~cm}$-distance between the sample and the target, and with a substrate speed of rotation of $20 \mathrm{rpm}$. To perform comparative studies of the various sets of samples, as-deposited films of IP or IPP were also vacuum-annealed at $150^{\circ} \mathrm{C}$, for two hours. Each film's thickness was measured using a Dektak 8 surface profilometer (Veeco Instruments Inc.).

The microstructures of the sputtered ITO films on different substrates were investigated using X-ray diffraction (XRD) patterns collected by a Bruker diffractometer (D8 Advance) with Cu Ka radiation, $\lambda=1.5406 \AA$. The surface morphologies were observed using Scanning Electron Microscopy (SEM) techniques by FEI Nova-Nano (lateral surface imaging) and FEI
Quanta 600 (in-situ surface cracking images). The optical transmittance and the respective haze values of all the samples deposited on PET substrates were measured using a UV-Vis spectrophotometer equipped with an integrating sphere (Varian Cary5000) for wavelengths ranging 300 to $800 \mathrm{~nm}$. For cross-sectional studies, specimen was prepared by using FEI Helios G4 UX Focused Ion Beam (FIB)/SEM dual-beam system equipped with a $\mathrm{Ga}+$ ion source. Pt layers were deposited on the surface region of interest by Electron and lon beam for specimen protection. Cross-section images were acquired on the same equipment using $5 \mathrm{kV}$ Electron beam with Inlens (TLD) detector.

Environmental studies of the various sets of samples were carried out using a TENNEY Environmental Test Chamber, in order to compare their relationships between electrical sheet resistance and relative humidity $(\mathrm{RH})$, at fixed chamber temperature. Samples were placed inside a humidity-controlled and temperature-controlled chamber, set at $80 \% \mathrm{RH}$ and $50{ }^{\circ} \mathrm{C}$; the time of exposure was kept to two hours. Electrical sheet resistance values were measured both before and after exposure to harsh conditions. Automated chamber values (temperature \& RH) were crossconfirmed every time, using a separate sensor connected to and within the chamber (fixed, close to the surface of the sample).

\section{Micro-tensile testing}

Straight rectangular samples $(80 \times 10 \mathrm{~mm})$ were obtained from films coated on 5" PET substrates.

The following two series of tests were performed, using an Instron 5944 Universal Testing Machine (UTM):

a. Monotonic tensile tests with a displacement rate of $1 \mathrm{~mm} / \mathrm{min}$ during which we monitored the applied load (macroscopic strain), the crack density, as well as changes in the electrical resistance of the samples. These tests allowed us to identify a meaningful loading window for subsequent cyclic tests, 2-point probe measurements were used to measure the in-situ resistance of film during loading. In that case, linear electrodes (copper wires attached with Silver Paste) were placed on the coated side of the thin film samples. Electrodes were connected to an U2741A digital multimeter (Agilent Technologies) to measure changes in the electrical resistance, over a $30 \mathrm{~mm}$ gauge length.

b. Loading/unloading tensile tests performed while monitoring the same variables as in (a) to evaluate any crack closure effects on the electrical sheet resistance of the thin film samples. Tests were divided into multiple incremental loading/unloading cycles in order to have a maximum extension of $10 \%$. After reaching a maximum extension for each cycle, samples were unloaded to measure their residual electrical resistance. All sets of thin film samples were tested to confirm the reproducibility of the experiments. Four-point probe measurements were used to measure to electrical resistance of unloaded film. These measurements were performed using Advanced Instrument technology (CMT Series), with a probe spacing of $1 \mathrm{~mm}$.

All tests were performed in a controlled environment, with temperature kept at $25^{\circ} \mathrm{C}$ and relative humidity (RH) at $65 \% \mathrm{RH}$. 
Optical images were obtained for a region of interest located at the center of the specimen, using a Zeiss microscope. Digital images were used to track the number of cracks during tests, and to evaluate the applied macroscopic strain. All in-situ microscopic images of various specimens were captured under controlled applied micro-tensile strain, using a specialized Kammrath and Weiss tool (1 kN Tensile Module, GmbH Germany).

\section{DATA AVAILABILITY}

The datasets generated during and/or analyzed during the current study are available from the corresponding author on reasonable request.

\section{ACKNOWLEDGEMENTS}

The research reported in this publication was supported by funding from King Abdullah University of Science and Technology (KAUST), under award number BAS/1/ 1315-01-01. The authors are thankful to Natanael Bolson and Dr. Jian Zhou from our COHMAS Laboratory, KAUST who assisted us with the preparation of the samples. Many thanks also to Ulrich Buttner for helping with the laser cutting, and his general assistance at the spin-coating facilities of Microfluidics Laboratory at KAUST. Thanks to Dr. Miaoxiang Chen and Dr. Wong Kim Chong for their support with the MVD and sputtering tools, respectively, at Nanofabrication Core Laboratory, KAUST.

\section{AUTHOR CONTRIBUTIONS}

G.L. conceptualized the study. Both G.L. and D.S. drafted this manuscript. D.S. carried out all the experiments, and R.T. helped with in-situ SEM measurements.

\section{ADDITIONAL INFORMATION}

Supplementary Information accompanies the paper on the npj Flexible Electronics website (https://doi.org/10.1038/s41528-019-0054-4).

Competing interests: The authors declare no competing interests.

Publisher's note: Springer Nature remains neutral with regard to jurisdictional claims in published maps and institutional affiliations.

\section{REFERENCES}

1. Rogers, J. A., Someya, T. \& Huang, Y. Materials and mechanics for stretchable electronics. Science 327, 1603-1607 (2010).

2. Masis, M. M., Wolf, S. D., Robinson, R. W., Ager, J. W. \& Ballif, C. Transparent electrodes for efficient optoelectronics. Adv. Electron. Mater. 3, 1600529-1600546 (2017).

3. Kang, H., Jung, S., Jeong, S., Kim, G. \& Lee, K. Polymer-metal hybrid transparent electrodes for flexible electronics. Nat. Commun. 6, 6503-6510 (2016).

4. Münzenrieder, N. et al. Stretchable and conformable oxide thin-film electronics. Adv. Electron. Mater. 1, 1400038-1400045 (2015).

5. Guo, X. et al. Highly conductive transparent organic electrodes with multilayer structures for rigid and flexible optoelectronics. Sci. Rep. 5, 10569-10578 (2015).

6. Zamarayeva, A. M. et al. Flexible and stretchable power sources for wearable electronics. Sci. Adv. 3, e1602051-e1602061 (2017).

7. Ginley, D. S, Hosono, H. \& Paine, D. C. Handbook of Transparent Conductors (Springer, New York, 2010).

8. Chopra, K. L., Major, S. \& Pandya, D. K. Transparent conductors-A status review. Thin Solid Films 102, 1-46 (1983).

9. Lippens, P., Muehlfeld, U., Chen J., Cranton, W. \& Finn, M. (eds.) Handbook of Visual Display Technology (Springer, Berlin, Heidelberg, 2012).

10. Burke, D. J. \& Lipomi, D. J. Green chemistry of green solar cells. Energy Environ. Sci. 6, 2053-2066 (2013)

11. Hecht, D. S., Hu, L. \& Irvin, G. Emerging transparent electrodes based on thin films of carbon nanotubes, graphene, and metallic nanostructures. Adv. Mater. 23, 1482-1513 (2011)
12. Kim, C. L., Jung, C. W., Oh, Y. J. \& Kim, D. E. A highly flexible transparent conductive electrode based on nanomaterials. NPG Asia Mater. 9, e438-e447 (2017).

13. $\mathrm{Xu}, \mathrm{Y}$. \& Liu, J. Graphene as transparent electrodes: fabrication and new emerging applications. Small 12, 1400-1419 (2016).

14. Wang, B. Y. et al. Enhanced light scattering and trapping effect of Ag nanowire mesh electrode for highly efficient flexible organic solar cell. Small 11, 1905-1911 (2015).

15. Yoo, J. H. et al. Silver nanowire-conducting polymer-ITO hybrids for flexible and transparent conductive electrodes with excellent durability. ACS Appl. Mater. Interfaces 7, 15928-15934 (2015).

16. Sannicolo, T. et al. Metallic nanowire-based transparent electrodes for next generation flexible devices: a review. Small 12, 6052-6075 (2016).

17. Cao, W., Li, J., Chen, H. \& Xue, J. Transparent electrodes for organic optoelectronic devices: a review. J. Photon. Energy 4, 040990-041018 (2014).

18. Vosgueritchian, M., Lipomi, D. J. \& Bao, Z. Highly conductive and transparent PEDOT:PSS films with a fluorosurfactant for stretchable and flexible transparent electrodes. Adv. Funct. Mater. 22, 421-428 (2012).

19. Wang, Y. et al. Highly stretchable, transparent, and conductive polymer. Sci. Adv. 3, e1602076-e1602086 (2017).

20. Cairns, D., Richard, R., Sparacin, D., Sachsman, S. \& Paine, D. Strain-dependent electrical resistance of tin-doped indium oxide on polymer substrates. Appl. Phys. Lett. 76, 1425-1427 (2000).

21. Leterrier, Y. et al. Mechanical integrity of transparent conductive oxide films for flexible polymer-based displays. Thin Solid Films 460, 156-166 (2004).

22. Leterrier, Y., Pinyol, A., Rougier, L., Waller, J. H. \& Manson, J. A. Electrofragmentation modeling of conductive coatings on polymer substrates. J. Appl. Phys. 106, 113508-113513 (2009).

23. Kim, E. H., Yang, C. W. \& Park, J. W. The crystallinity and mechanical properties of indium tin oxide coatings on polymer substrates. J. Appl. Phys. 109, 043511-043518 (2011).

24. Park, J. W., Kim, G., Lee, S. H., Kim, E. H. \& Lee, G. H. The effect of film microstructures on cracking of transparent conductive oxide coatings on polymer substrates. Surf. Coat. Technol. 205, 915-921 (2010).

25. Saleh, M. N. \& Lubineau, G. Understanding the mechanisms that change the conductivity of damaged ITO-coated polymeric films: A micro-mechanical investigation. Sol. Energy Mater. Sol. Cells 130, 199-207 (2014).

26. Sun, K. et al. Review on application of PEDOTs and PEDOT:PSS in energy conversion and storage devices. J. Mater. Sci: Mater. Electron 26, 4438-4462 (2015).

27. Greco, F. et al. Ultra-thin conductive free-standing PEDOT/PSS nanofilms. Soft Matter 7, 10642-10650 (2011).

28. Lipomi, D. J. et al. Electronic eroperties of transparent conductive films of PEDOT: PSS on stretchable substrates. Chem. Mater. 24, 373-382 (2012).

29. Zhou, J. et al. The temperature-dependent microstructure of PEDOT/PSS films: insights from morphological, mechanical and electrical analyses. J. Mater. Chem. C. 2, 9903-9910 (2014).

30. Tsukruk, V. V. \& Bliznyuk, V. N. Adhesive and friction forces between chemically modified silicon and silicon nitride surfaces. Langmuir 14, 446-455 (1998).

31. Howarter, J. A. \& Youngblood, J. P. Optimization of silica silanization by 3aminopropyltriethoxysilane. Langmuir 22, 11142-11147 (2006).

32. Cullity, B. D. \& Stock, S. R. Elements of X-ray Diffraction. 3rd edn. 98-99. (PrenticeHall, New Jersey, 2001).

Open Access This article is licensed under a Creative Commons Attribution 4.0 International License, which permits use, sharing, adaptation, distribution and reproduction in any medium or format, as long as you give appropriate credit to the original author(s) and the source, provide a link to the Creative Commons license, and indicate if changes were made. The images or other third party material in this article are included in the article's Creative Commons license, unless indicated otherwise in a credit line to the material. If material is not included in the article's Creative Commons license and your intended use is not permitted by statutory regulation or exceeds the permitted use, you will need to obtain permission directly from the copyright holder. To view a copy of this license, visit http://creativecommons. org/licenses/by/4.0/.

(c) The Author(s) 2019 